\title{
MOTA Syndrome: Molecular Genetic Confirmation of the Diagnosis in a Newborn with Previously Unreported Clinical Features
}

\author{
D. Mitter ${ }^{a} \quad$ D. Schanze ${ }^{d} \quad$ I. Sterker ${ }^{b} \quad$ D. Müller ${ }^{a} \quad$ H. Till ${ }^{c} \quad$ M. Zenker ${ }^{d}$ \\ ${ }^{a}$ Institute of Human Genetics, b Department of Ophthamology and ${ }^{\mathrm{c}}$ Department of Pediatric Surgery, University

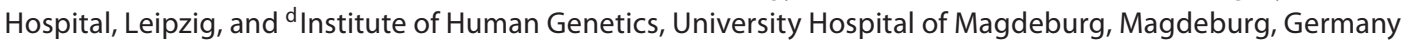

\section{Key Words}

Compound heterozygous mutations $\cdot$ FREM1 gene $\cdot$ MOTA syndrome

\begin{abstract}
MOTA syndrome, the acronym for Manitoba-oculo-trichoanal syndrome (OMIM 248450), is a distinct autosomal recessive multiple malformation syndrome caused by mutations in the FREM1 gene (OMIM 608944). Eight patients with MOTA syndrome and a pathogenic FREM1 mutation have previously been documented. We report on a new male patient, 3.5 months old, with MOTA syndrome, who presented with the following features: bilateral incomplete cryptophthalmos with a completely fused, ill-defined upper eyelid and a keratinized cornea, hypertelorism, a broad tip of the nose, a circle-shaped whirl of hair on the forehead, and a low anorectal malformation, which could be corrected on day 2 of life without a colostomy. In expansion to the previously reported phenotype of MOTA syndrome, the patient showed characteristic features reported in patients with Fraser syndrome, including dysplastic ears, cutaneous syndactyly $3 / 4$ of the hands and syndactyly $2 / 3$ of the right foot. Molecular analysis of FREM1 identified compound heterozygosity for a new frameshift deletion in exon 24 (c.4629delC, p.F1544SfsX62) and a previously reported missense mutation in exon 21
\end{abstract}

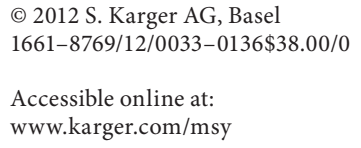

(c.3971T>G, p.L1324R). This report further extends the phenotype of MOTA syndrome and underscores the overlapping clinical spectrum of FRAS-FREM complex diseases.

Copyright $\odot 2012$ S. Karger AG, Basel

Manitoba-oculo-tricho-anal (MOTA) syndrome is a rare autosomal recessive disorder characterized by eye defects including eyelid coloboma, cryptophthalmos, anophthalmia/ microphthalmia, abnormal hair growth from scalp to eyebrow, bifid or broad nasal tip, and gastroinstestinal anomalies including omphalocele and anorectal malformations. Recently, homozygous and compound heterozygous mutations in the FREM1 gene (OMIM 608944), located on chromosome 9p22.3, were found to cause MOTA syndrome [Slavotinek et al., 2011]. Homozygous loss of function mutations in FREM1 had been found previously in patients with BNAR syndrome (Bifid nose with or without anorectal and renal anomalies) [Alazami et al., 2009], thus demonstrating that MOTA and BNAR syndromes are allelic conditions. The phenotypes of these conditions overlap with Fraser syndrome caused by mutations in FRAS1, FREM2 and GRIP1

D. Mitter and D. Schanze contributed equally to this work. 
Fig. 1. Facial phenotype. a Patient at the age of 6 days showing bilateral incomplete cryptophthalmos with keratinized cornea, hypertelorism, a wide nasal bridge and tip of the nose, and a large mouth with a small upper lip. Note circle-shaped whirl of hair on the left forehead, growing into the eyebrow. $\mathbf{b}$ The patient at the age of 3.5 months with surgically repaired eyelid coloboma. Note dysplastic ears.
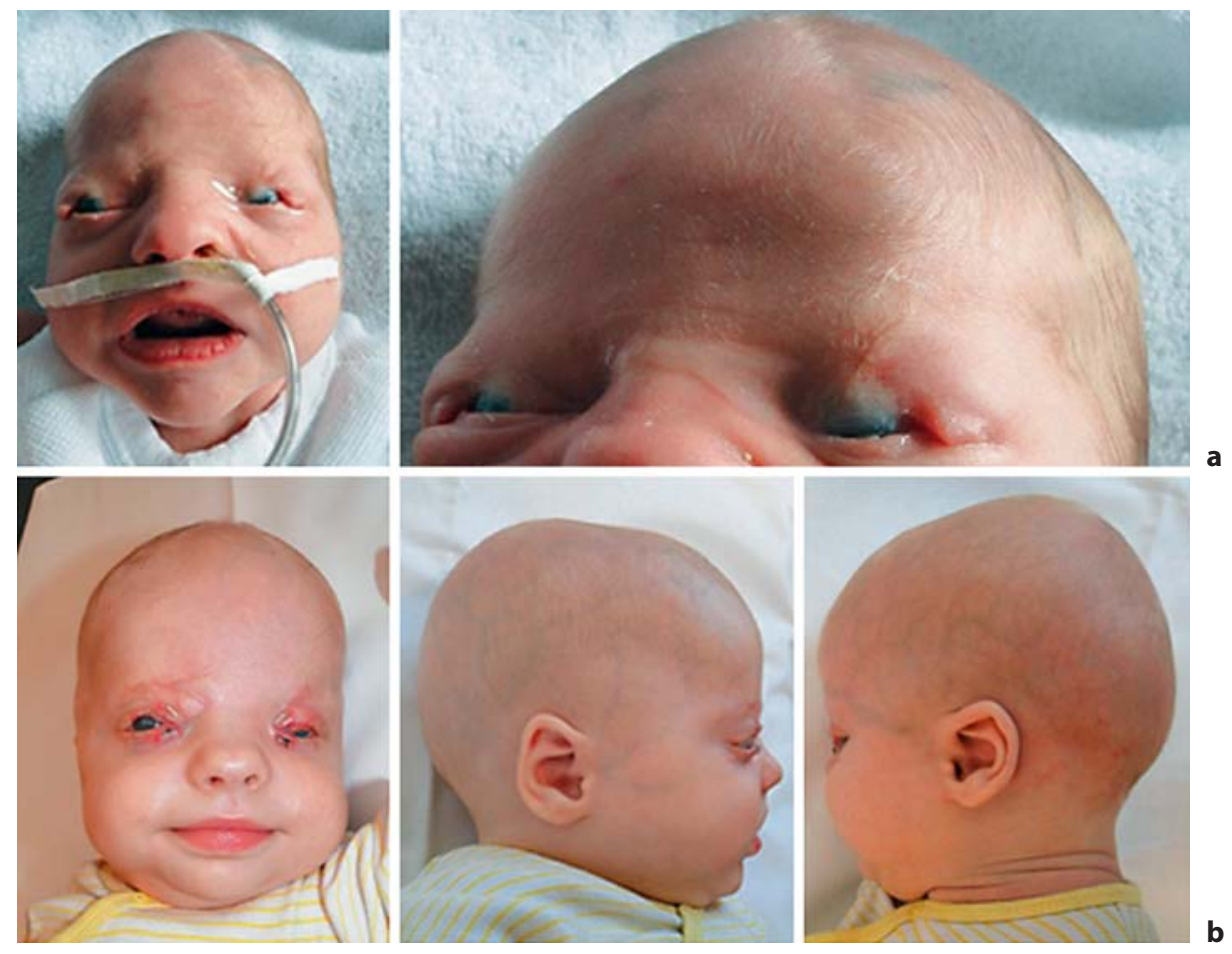

[Slavotinek and Tifft 2002; van Haelst et al., 2007, 2008; Vogel et al., 2012]. Fraser, BNAR and MOTA syndrome are therefore considered to constitute a group of clinically and pathogenetically related FRAS-FREM complex diseases with the most severe phenotype seen in patients with Fraser syndrome (cryptophthalmos, syndactyly, ambigious genitalia, ear malformation, and renal defects).

During mouse embryonic development, Frem 1 has been shown to be widely expressed, especially in the dermis and differentiating epidermal structures, such as mammary and Meibomian glands, teeth, and hair follicles [Alazami et al., 2009; Vissers et al., 2011]. Strong Frem1 expression was further detected in the nose of the mouse and primarily in the epithelial-mesenchymal transitional region at the midline, suggesting an important role in fusion of the nasal processes during gestation [Alazami et al., 2009]. Further analysis of Frem1 mutant mice found evidence that Frem 1 is involved in craniofacial development similar to the facial and ocular phenotype as seen in humans and in anal development [Slavotinek et al., 2011].

Up to date, only 8 patients from 4 families with mutations of FREM1 and MOTA syndrome have been described [Slavotinek et al., 2011]. These patients showed
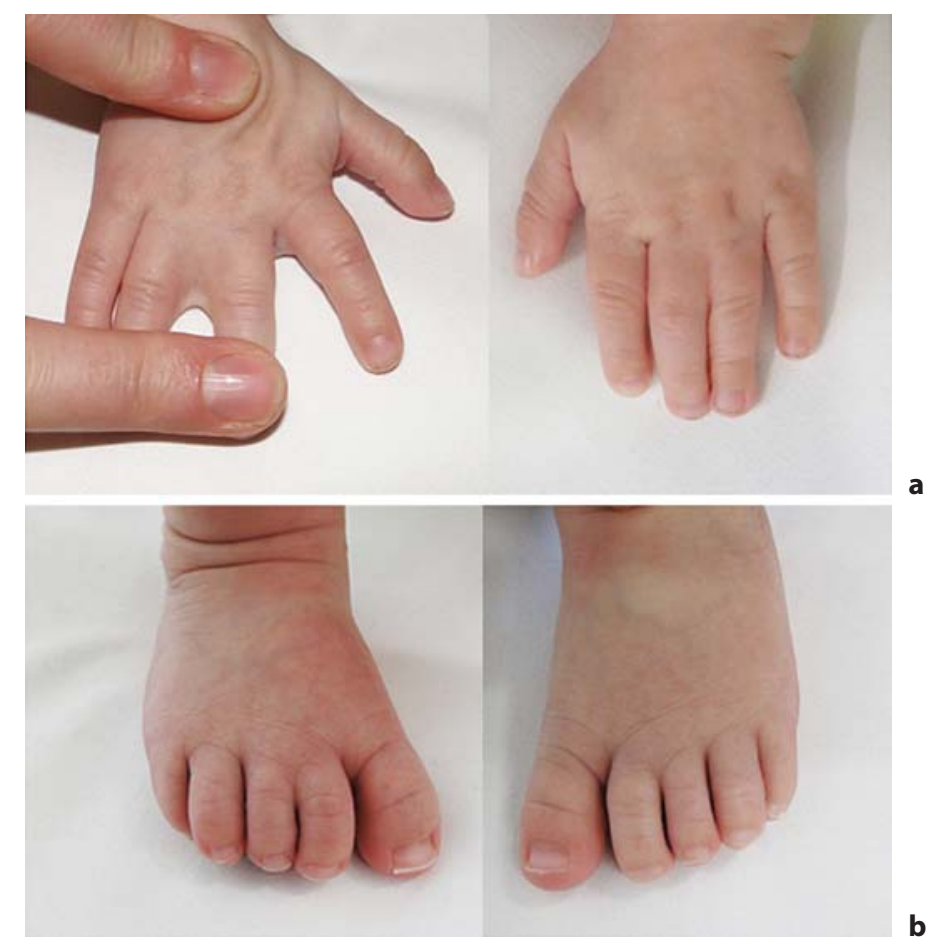

Fig. 2. Hands and feet of the patient at the age of 3.5 months. a Cutaneous syndactyly $3 / 4$ of both hands. b Syndactyly $2 / 3$ of the right foot. 
Fig. 3. Chromatograms for FREM1 exons 21 and 24 in a normal control, the patient and his parents. The patient shows 2 heterozygous FREM1 alterations: c.3971T>G in exon 21, also present in his mother (heterozygous), and c.4629delC in exon 24, also present in his father (heterozygous).
Exon21

control

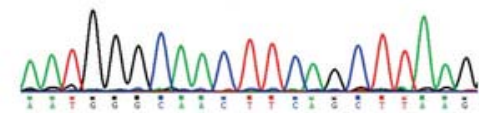

patient

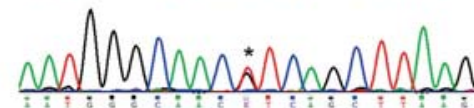

mother

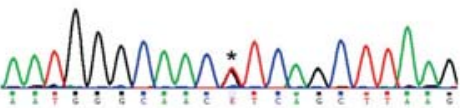

father
Exon24
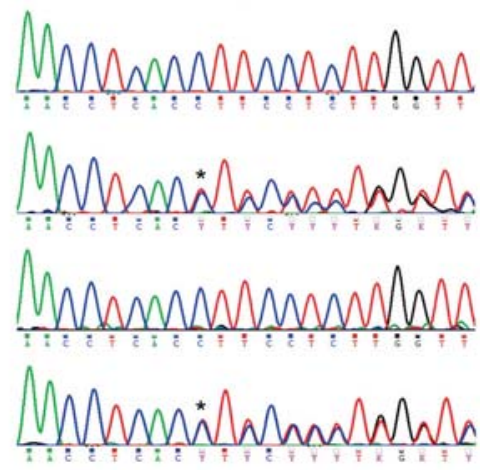

high interfamilial and intrafamilial variability of the phenotype. Here we report on a new patient with MOTA syndrome, in whom the clinical diagnosis could be confirmed by demonstration of compound heterozygosity for 2 FREM1 mutations.

\section{Case Report}

This boy is the first child born to German parents, a 26-yearold woman and a 29 -year-old man. The parents are not consanguineous. No fetal abnormalities were recorded during pregnancy. The boy was born after $39+3$ weeks of pregnancy via caesarean section with a birth weight of $3,480 \mathrm{~g}(-0.1 \mathrm{SD})$, length $52 \mathrm{~cm}$ (mean) and head circumference $36 \mathrm{~cm}(+0.6 \mathrm{SD})$. Apgar score was 10/10. At birth, bilateral incomplete cryptophthalmos, cutaneous syndactyly $3 / 4$ of both hands, syndactyly $2 / 3$ of the right foot were noted (fig. 1, 2). In addition, an imperforate anus with a perineal fistula was noticed. Since the defect could be graded as a low anorectal malformation, the child underwent a primary repair by posterior sagital anorectoplasty at day 2 of life. The postoperative course was completely uneventful.

Ophthalmic surgery of the bilateral eyelid malformation was performed at day 10 of life to construct eyelids with mucous membrane-lined fornices. The upper eyelids were created by a $6-\mathrm{mm}$ free tarso-conjunctival graft harvested from the temporal part of the lower very lax eyelid. The free tarso-conjunctival flap was covered by a skin rotation flap from the upper eyelid skin. A free 10$\mathrm{mm}$-long conjunctival graft from the inferior conjunctival fornices was transplanted to the bare sclera and to form the upper conjunctival sac. The postoperative period was without complication. Three months after surgery the child was able to close his eyes while sleeping and crying. A permanent application of lubricant ointment was necessary.

The visual function showed perception of light and moving objects.
At the age of 2 months, he reached normal measurements with a height of $59 \mathrm{~cm}(+1 \mathrm{SD})$, weight $4,640 \mathrm{~g}(-0.4 \mathrm{SD})$ and head circumference $39 \mathrm{~cm}$ (mean). He showed hypertelorism, downslanting palpebral fissures, a broad nasal bridge and tip of the nose, dysplastic ears, a circle-shaped whirl of hair on the left forehead, and a naevus flammeus on the scalp and neck (fig. 1). His genitalia were normal. He showed normal motor development. Kidney ultrasound examination showed mild pyelectasis on the left kidney. BERA testing at the age of 3.5 months confirmed normal hearing.

Taken together, the clinical phenotype suggested a FRASFREM complex disorder. Due to the absence of renal malformations and the type of ocular anomalies, our provisional clinical diagnosis was MOTA rather than Fraser syndrome.

\section{Genetic Analysis}

With appropriate informed consent of the parents, blood was obtained from the patient and both parents. Conventional chromosome analysis in the patient revealed a normal male karyotype.

The entire coding (exons 1-36) and flanking intronic regions of FREM1 were amplified by PCR according to standard procedures. PCR amplicons were purified and directly sequenced using the BigDye Terminator Cycle Sequencing kit (version 1.1, Applied Biosystems, Foster City, Calif., USA) and analyzed in an automated capillary sequencer (Applied Biosystems). Primer sequences are available on request. Sequence data was processed using ABI software and analyzed using the Sequence Pilot Software (JSI medical systems). DNA sequences were compared to the FREM1 reference sequence deposited in the public database (GenBank accession code NM_144966).

Analysis of FREM1 in the patient identified a heterozygous missense mutation in exon 21 (c.3971T $>$ G) predicting a replacement of leucine by aginine (p.L1324R) and a heterozygous frameshift mutation in exon 24 (c.4629delC) predicted to lead to a premature stop p.F1544SfsX62 (fig. 3). Sequencing of FREM1 exon 21 in both parents revealed heterozygosity for the c.3971T $>$ G sequence variant (predicting p.L1324R) in the mother, whereas the father was carrier of the frameshift mutation in exon 24 . 


\section{Discussion}

MOTA syndrome is clinically and pathogenetically related to, but distinct from Fraser syndrome. In both syndromes, ocular abnormalities of the cryptophthalmos spectrum occur. These range from shortened eyelids attached to the surface of the bulbus to the complete coverage of the eyeball with skin. An extended hairy wedge over the forehead to the ocular region may be present. It appears that complete cryptophthalmos is less common in MOTA syndrome. Milder eyelid abnormalities, including incomplete cryptophthalmos with an ill-defined upper eyelid completely fused combined with an abnormal globe and a keratinized cornea as seen in the present case may be more typical. The variability of ocular anomalies in MOTA syndrome is wide and a clear genotype phenotype relationship has not yet been established. Yet, eyes with incomplete cryptophthalmos, as seen in the present case, represent a fundamental failure in ocular development and have a poor prognosis for vision.

Further overlapping features of MOTA and Fraser syndrome include nasal (bifid nose), umbilical (omphalocele) and anorectal anomalies (imperforate anus, dis- placed anus). The previously published observations of MOTA syndrome do not show laryngotracheal and renal anomalies or syndactyly, which are frequent in Fraser syndrome. It seems obvious that the absence of particularly laryngotracheal atresia and bilateral renal agenesis is related to a much better survival rate in MOTA as compared to Fraser syndrome. Despite these clear differences, the presented patient emphasizes the clinical overlap between Fraser and MOTA syndromes by showing that syndactyly and ear anomalies may occur also in MOTA syndrome. This suggests that no single clinical feature might distinguish MOTA from Fraser syndrome. Further information on this question can be expected from molecular genetic testing to aid in the diagnosis of such cases.

\section{Acknowledgements}

We wish to thank the patient and his family for their cooperation. We thank Professor Eberhard Passarge for valuable discussion and reading the manuscript. We also thank Professor Wolfgang E. Fleig and the Executive Board of the University of Leipzig Hospitals and Clinics for continuous support.

\section{References}

Alazami AM, Shaheen R, Alzahrani F, Snape K, Saggar A, et al: FREM1 mutations cause bifid nose, renal agenesis, and anorectal malformations syndrome. Am J Hum Genet 85: 414-418 (2009).

Slavotinek AM, Tifft CJ: Fraser syndrome and cryptophthalmos: review of the diagnostic criteria and evidence for phenotypic modules in complex malformation syndromes. J Med Genet 39:623-633 (2002).
Slavotinek AM, Baranzini SE, Schanze D, Labelle-Dumais C, Short KM, et al: Manitobaoculo-tricho-anal (MOTA) syndrome is caused by mutations in FREM1. J Med Genet 48:375-382 (2011)

van Haelst MM, Scambler PJ; Fraser Syndrome Collaboration Group, Hennekam RC: Fraser syndrome: a clinical study of 59 cases and evaluation of diagnostic criteria. Am J Med Genet A 143A:3194-3203 (2007).

van Haelst MM, Maiburg M, Baujat G, Jadeja S, Monti E, et al: Molecular study of 33 families with Fraser syndrome new data and mutation review. Am J Med Genet A 146A:22522257 (2008).
Vissers LE, Cox TC, Maga AM, Short KM, Wiradjaja F, et al: Heterozygous mutations of FREM1 are associated with an increased risk of isolated metopic craniosynostosis in humans and mice. PLoS Genet 7:e1002278 (2011).

Vogel MJ, van Zon P, Brueton L, Gijzen M, van Tuil MC, et al: Mutations in GRIP1 cause Fraser syndrome. J Med Genet 49:303-306 (2012). 